\title{
Processo de planejamento para produção, execução e avaliação de conteúdos crossmidiáticos, educativos e televisivos
}

\author{
Rafaela Ponte Lisboa Cardoso- LUQS/UNIFOR, Lab. Multimeios/UFC- \\ rafaela@multimeios.ufc.br \\ Maria Elizabeth Sucupira Furtado - LUQS/UNIFOR _elizabet@unifor.br \\ Hermínio Borges Neto - Lab.Multimeios/UFC - hermínio@multimeios.ufc.br
}

\section{RESUMO}

O avanço do Sistema Brasileiro de Televisão Digital interativa (SBTVDi) vem ampliando as perspectivas de utilização da televisão no contexto educacional, em virtude das possibilidades de interatividade e comunicação. Assim, começa-se a discutir acerca do desenvolvimento de conteúdos educativos que atendam as necessidades dessa nova realidade. Neste artigo pretende-se descrever orientações para a produção de materiais educativos para a Televisão Digital interativa (TVDi). Apresentar-se-á um processo de planejamento para produção, execução e avaliação de conteúdos educativos, que agrega dimensões tecnológicas, comunicacionais e educacionais, e uma metodologia pedagógica, Sequência Fedathi, que visa auxiliar na definição da abordagem do conteúdo a ser apresentado.

Palavras-chave: Televisão digital. Sequência Fedathi. Conteúdos educativos.

\section{Process for production planning, implementation and evaluation of contents crossmidiáticos, educational television}

\begin{abstract}
The improvement of the Brazilian System of Interactive Digital Television (iDTV) has amplified usage perspectives for television in the educational realm, due to new possibilities of interaction and communication. Thus, there is a growing discussion over the development of educational contents that will address the needs of this new reality. The present article intends to describe guidelines for the production of educational materials for iDTV. A process will be presented for production planning, execution and evaluation of educational contents which aggregates technological, communicational and educational dimensions, as well as a pedagogical methodology, Fedathi Sequence, that aims to support the approach to content that will be presented.
\end{abstract}

Keywords: Digital television. Following Fedathi. Educational content. Content Production.

\section{INTRODUÇÃO}

A utilização da Televisão como instrumento de educação no Brasil iniciou na década de 60, por meio de iniciativas de instituições educacionais e governamentais, promovendo o desenvolvimento de projetos, como o surgimento de TVs Educativas em vários Estados brasileiros. Tal iniciativa surgiu, em virtude da televisão no mercado brasileiro chegar a aproximadamente $96 \%$ da população (Cruz, 2008), oferecendo um sistema próprio, aberto e gratuito. Portanto, o acesso universal à educação via TV sempre se apresentou como uma possibilidade viável neste cenário deficitário. 
Diante desta perspectiva, inicia-se uma vertente de Ensino a Distância (EaD), que se amplia com o desenvolvimento de diversos meios de transportes e comunicação. Especificamente, em 2000, iniciaram os esforços na busca de soluções para a aprendizagem a distância via web, onde os alunos passaram a trabalhar de forma colaborativa, e os diversos formadores, que acompanhavam tais alunos, passaram a ter maior autonomia e a serem coautores dos conteúdos disponíveis nos sistemas computacionais.

Com o surgimento do SBTVDi em 2007, a TV volta a ser um meio promissor para propiciar a EaD. Vale salientar que, soluções comerciais de sistemas a cabo ou satélite são privadas e pagas, incompatíveis com o SBTVDi que é terrestre e gratuito. Esse sistema se sobressai por ser uma solução brasileira, contendo um middleware chamado GINGA (Ginga, 2011) capaz de interpretar alternativas de linguagens de programação para implantar a interatividade em diversos dispositivos. A implantação da TVDi no Brasil está acontecendo gradativamente. Destaca-se uma série de investimentos governamentais financiando projetos realizados por empresas, emissoras e/ou universidades. Dentre eles, está o projeto que originou esta pesquisa, que visa o ensino da matemática via TVDi (Lisboa, 2011).

Contrariamente ao que tem sido feito neste projeto, trabalhos (Arbex; Sens; Spanhol, 2012) (Sacrini, 2006) que visam a EaD não abrangem toda a cadeia de valor (desde a concepção de um curso até a sua recepção pelos alunos) não trazendo soluções integradas para a produção de conteúdos com relação aos aspectos educativo, comunicativo e tecnológico. Além disto, eles apresentam o uso do padrão brasileiro, mas em ambientes simulados (como usando emuladores para simularem a interatividade nos aparelhos de TV) (Sacrini, 2006). Faltam iniciativas que apontem como tratar a interatividade (como de personalização, de usabilidade), explorando os diversos recursos disponíveis por este meio de comunicação (como a sua convergência com outros dispositivos, ter dados na TVDi, além de áudio e vídeo, etc.) para ampliar as experiências de uso com a TVDi.

Este artigo apresenta orientações que podem ser seguidas para nortear uma cadeia produtiva desde o planejamento da produção de conteúdos educativos televisivos, até a execução e avaliação dos conteúdos produzidos nos dispositivos alvos.

Este trabalho está organizado da seguinte maneira, primeiramente descrevem-se alguns trabalhos relacionados à produção de conteúdo. Na seção 3, destacam-se os objetos de estudo e a questão de pesquisa deste projeto. Enquanto que na seção 4, apresenta-se o contexto onde este trabalho se desenvolveu. Posteriormente, as fases do processo de desenvolvimento de conteúdos e experiências com o desenvolvimento de objetos de aprendizagem são descritos. Por fim, ressaltam-se os resultados e implicações, seguidos de uma discussão e conclusão.

\section{TRABALHOS RELACIONADOS}

Alguns trabalhos acerca dos usos da TVDi para aprendizagem com foco na produção e no desenvolvimento de conteúdos educativos vêm sendo desenvolvidos. Belda e Da Silva (2013), por exemplo, indicam diretrizes de produção de conteúdo para Televisão Digital com foco na difusão do conhecimento por meio de programas de Educação à Distância.

Belda (2010), em sua tese de doutorado, apresenta modelos estruturais de conteúdos educativos para TVDi são propostos com o intuito de fornecer subsídios para processos colaborativos de produção de conteúdos em redes e comunidades de aprendizagem associadas a canais universitários e educativos de televisão. São modelos 
que estruturam uma narrativa de um programa audiovisual, formatado para os padrões da TVDi, bem como oferecem uma proposta de qualificação do ambiente digital, a citar, tem-se a proposta descrita em Castro e Freitas (2011).

Já Roesler et al. (2010) apresentam, em seus estudos, um novo modelo para utilização da televisão digital em educação, onde o telespectador tem a possibilidade de acessar listas de cursos educacionais. Os autores validam o modelo com a implementação do curso educacional "Viva Saudável".

Arbex, Sens e Spanhol (2012) trazem possibilidades de desenvolvimento de conteúdos educacionais para TVDi e EaD, e mostram os resultados de alguns exemplos da TV Escola, do uso da linguagem audiovisual e de interfaces gráficas.

Estas pesquisas são relevantes, principalmente porque sugerem perspectivas diferentes de trabalhar os aspectos tecnológicos, comunicacionais a educacionais na TVDi (Castro e Freitas, 2011),(Arbex; Sens; Spanhol, 2010), mas o fazem de forma isolada (Belda e Da Silva, 2013). Entende-se que poucos são os que têm voltado suas pesquisas para a produção integrada de conteúdos trazendo orientações que norteiem profissionais na definição e avaliação de cenários interativos de aprendizagem, ampliando os usos dos conteúdos educativos para além da própria TVDi. A pesquisa apresentada neste artigo apresenta um processo de planejamento de produção de conteúdos educativos televisivos e sua exploração em cenários convergentes de ensino da matemática.

\section{OBJETIVO DO ESTUDO E QUESTÃO DA PESQUISA}

O objetivo deste estudo é apresentar um processo de planejamento para produção, execução e avaliação de conteúdos educativos para TVDi, bem como os resultados obtidos em cenários de aprendizagem de temas de matemática destinados a estudantes da rede pública de ensino de Fortaleza, Ceará, Brasil.

Foram produzidos conteúdos educativos por professores, transmitidos e acessados pelo telespectador aluno (usuário) no formato fullseg via a TVDi e oneseg, recepção via celular. Um conteúdo é chamado T-VILO (Matos;Furtado, 2012), conceito oriundo do conceito de VILO - Video Interactive Learning Objects, em português: Objeto de Aprendizagem em Vídeo Interativo, que é uma evolução da noção objeto de aprendizagem tradicional. Um T-VILO é composto de: 1) um vídeo e; 2) conteúdos extras e formados por várias mídias, como, textos, questionários, imagens. Um T-VILO produzido a partir do processo proposto está imbuído de aspectos que atendem a duas perspectivas: i) a de convergência digital com a TVDi, que visa a transacionar conteúdos extras da TVDi para outros dispositivos, como o celular e; ii) a de conteúdo crossmídia, que visa a produção de conteúdos de forma diferenciada e em função do dispositivo de interação alvo.

A questão desta pesquisa é a seguinte: Como guiar profissionais a desenvolverem conteúdos interativos televisivos (os T-VILOs), que levem os telespectadores aluno a interagirem usufruindo das possibilidades de uso da TVDi? Essa questão será respondida durante a descrição do processo proposto.

Os resultados esperados com esse processo são: i) roteiros interativos de T-VILOs planejados a partir dos interesses dos usuários e desenvolvidos com recursos que cubram os aspectos tecnológicos, comunicacionais e educacionais de forma integrada e; ii) cenários de aprendizagem e de interação com esses conteúdos, via as aplicações interativas, que são transmitidas e avaliadas. Antes de descrever esse processo, serão descritos os recursos previamente desenvolvidos e considerados nesta pesquisa. 


\section{CONTEXTO DA PESQUISA}

Anteriormente a esta pesquisa, decisões metodológicas e comunicacionais foram tomadas para a produção de um T-VILO e recursos tecnológicos necessários para obtêlos na TVDi e no celular foram desenvolvidos. Essas decisões e recursos, que embasaram a descrição do processo de planejamento descrito neste artigo, se referem a três categorias, são elas:

- Tecnológica, se refere à aplicação desenvolvida para a produção de um T-VILO, à infraestrutura de transmissão dos conteúdos, e à aplicação que irá permitir o acesso aos conteúdos transmitidos (Matos e Furtado, 2011) (Matos, 2011). A primeira aplicação, chamada T-VILO Maker, suporta a geração de conteúdos multimídia e extras, como questionários, informações acerca do conteúdo apresentado no vídeo. Essa aplicação, que funciona na plataforma Web, empacota os conteúdos produzidos com a segunda aplicação, chamada T-VILO Viewer, responsável pela exibição do conteúdo na TVDi ou no celular (Celes, 2011);

- Pedagógica, se refere à aplicação da metodologia pedagógica, chamada Sequência Fedathi (SF) (Borges Neto; Santana, 2003), escolhida para nortear o desenvolvimento do vídeo e dos conteúdos em multimídia (Lisboa et al.,2010) criados no T-VILO Maker. Essa metodologia tem como base a aprendizagem por resolução de problemas explorados, colocando o aluno em uma posição ativa no processo de ensinoaprendizagem, tendo a possibilidade de interagir e intervir diante das informações apresentadas. Sua aplicação divide-se nas seguintes etapas: tomada de posição, maturação ou debruçamento, solução e prova. A SF vem sendo pesquisada para diversos ambientes educativos, como: educação básica, modalidade a Distância, produção de aulas e conteúdos para a WEB e para TVDi, a partir deste presente projeto sendo realizado. Na próxima seção, apresenta-se como a SF é proposta para ser aplicada na produção de um T-VILO e;

- Comunicacional, se refere ao processo de comunicação a ser estabelecido entre o telespectador aluno e o apresentador de um programa (de um vídeo) (Lisboa et al., 2011). Um processo de comunicação ocorre quando, em um determinado contexto, um emissor emite uma mensagem a um receptor por meio de um canal (Jakobson, 1960). Assim que o receptor recebe a mensagem, ele gera uma ideia daquilo que o emissor quis dizer e inicia o seu processo de compreensão (De Souza, 2005).

Convém ressaltar a existência de três equipes de profissionais: pedagógica, formada por pedagogo e produtor de conteúdo; técnica, composta por designer do TVILO e técnico em informática, e; produção, formada por cameraman e editor de vídeo.

A partir das categorias supracitadas e dos profissionais envolvidos, o processo de planejamento foi estruturado, o qual será explicado a seguir.

\section{FASES DO PROCESSO DE DESENVOLVIMENTO DE CONTEÚDOS}

Nesta seção, descreve-se as cinco fases do processo de planejamento para produção, execução e avaliação de conteúdos educativos. Esse processo está baseado no de elaboração dos conteúdos televisivos do Telensino (Campos, 2005). O que os difere é a introdução do desenvolvimento de um roteiro para uma aplicação interativa, bem como a inserção das categorias tecnológicas, comunicacionais e pedagógicas que norteiam a elaboração dos roteiros dos vídeos.

\subsection{Fase de Produção: Desenvolvimento de um T-VILO}

Esta fase inicia com a definição do tema, do conteúdo que se irá trabalhar; em seguida, é traçado o perfil do usuário, observando seus interesses e sua afinidade acerca do assunto. Parte-se então para o desenvolvimento do roteiro, que deve ater-se à seguinte 
estrutura de três colunas: uma com informações sobre as cenas do vídeo; outra com o áudio, contendo o diálogo entre os personagens da trama e/ou fala do apresentador; e outra com a aplicação interativa, que possui informações sobre as telas da aplicação, como: questões, imagens e textos.

As cenas do vídeo são pensadas como uma trama a ser tratada nos diálogos travados pelos personagens. Aqui também devem ser definidos os objetivos pedagógicos, ou seja, o que os telespectadores aluno precisam apreender e/ou aprender após a exibição do vídeo. É na formulação do roteiro, que as equipes pedagógica, técnica e de produção devem seguir a metodologia SF tomando como base as quatro etapas mencionadas a seguir.

Primeiramente, estrutura-se a "tomada de posição", etapa na qual inicia a apresentação do conteúdo. Tal estruturação é feita por meio de uma pequena história que envolve dois personagens, que se deparam geralmente com situações-problema. $\mathrm{O}$ problema é apresentado ao telespectador aluno, de forma a possibilitar a relação entre a situação proposta e o saber que deve ser ensinado.

Posteriormente, tem-se a fase de “maturação ou debruçamento", que ocorre quando questionamentos são apresentados por dois personagens. Um problema visa favorecer o desenvolvimento dos raciocínios e argumentos dos alunos que assistem ao T-VILO. Nesta fase, o aluno precisa reconhecer os significados das conjecturas apresentadas na etapa anterior e a partir deste reconhecimento, gradativamente, trabalhar mais sobre o problema em questão. Em seguida, por meio da "solução", quando o aluno escolhe uma das respostas apresentadas, independentemente de estar certa ou errada, o T-VILO apresenta feedback às possíveis reflexões que facilitam o aluno a realizar comparações e a organizar ideias. Por fim, a etapa "prova” que apresenta a solução mais sistematizada, assim o T-VILO traz alguns conceitos, por meio da reflexão e apontamentos dos personagens que levantam os problemas planejados no vídeo. A partir deste momento, se estabelece as relações que envolvem o saber em questão e o seu processo de validação (Lisboa, 2011).

\subsection{Fases de realização e de supervisão}

Na fase de realização ocorre a gravação dos vídeos e o desenvolvimento dos conteúdos usando a aplicação T-VILO Maker. São estabelecidos os contatos com profissionais para a gravação dos vídeos e feitas a edição do vídeo e criação de vinhetas.

Em seguida é feita a seleção de atores que encenarão no vídeo. Eles precisarão compreender os pressupostos teóricos da SF, pois devem saber como passar as informações/questionamentos do conteúdo a ser abordado no vídeo de forma a suscitar a reflexão nos telespectadores. Para tanto, iniciam-se os ensaios e as gravações dos vídeos - que podem ser externas, ou seja, em um parque, shopping, cantina dentre outros; internas, dentro de um estúdio fechado (Kellison, 2007). Paralelamente, a equipe pedagógica age na produção do conteúdo extra/aplicação interativa, baseada no roteiro previamente definido na fase anterior, usando a aplicação T-VILO Maker.

É importante destacar que a consideração da perspectiva crossmidiática ocorre nesta fase. Para fazer uso desta possibilidade, o produtor deve inserir um endereço WEB no campo indicado no T-VILO Maker e a ferramenta gera um QRCode com tal conteúdo inserido. O QRCode é uma evolução dos códigos de barras bidimensionais que são amplamente utilizados em produtos industrializados e sua vantagem é a maior capacidade de armazenamento de dados (Celes, 2011). Objetiva-se assim a mobilidade, onde o conteúdo poderá ser visto em outro momento, desde que o usuário da TVD i tenha lido o QRCode e armazenado em seu dispositivo/ celular. 
$\mathrm{Na}$ fase de supervisão, os profissionais da equipe pedagógica e técnica se organizam para a verificação do material produzido, ou seja, do vídeo e do conteúdo desenvolvimento no T-VILO Maker. A equipe técnica verifica o formato do vídeo e realiza uma transmissão teste do conteúdo, visando averiguar a existência de falhas em parâmetros da conversão do áudio e vídeo.

A equipe pedagógica avalia o conteúdo gerado, transmitido e visualizado no TVILO Viewer, analisando a distribuição das informações na tela, a existência de algum erro de digitação e ou de falha na exibição de alguma imagem. Há também a necessidade de avaliar as possibilidades de ganho no processo de ensino-aprendizagem proporcionado pelo T-VILO, ou seja, se os telespectadores aluno, ao assisti-lo, aprendem acerca do conteúdo exibido e se são capazes de analisar aspectos de interatividade presentes nas aplicações interativas (Lisboa, 2011).

\subsection{Fases de recepção e de avaliação}

A fase de recepção envolve o momento em que a equipe pedagógica planeja os cenários de aprendizagem, em que os T-VILOs produzidos serão explorados. Um cenário deve ser escolhido visando auxiliar um professor na explicação de um conteúdo desejado e relacionado ao T-VILO. Estrutura-se um cenário de acordo com os objetivos de aprendizagem traçados durante a confecção do roteiro do T-VILO, e devendo ter uma duração de 40 a 50 minutos. Considere um cenário em uma sala de aula, por exemplo. Antes dos alunos assistirem ao T-VILO, um professor fala sobre o conteúdo a ser exibido e diz que poderá tirar dúvidas durante a exibição, fazendo, assim, uma sensibilização acerca dessa modalidade de curso na TVDi.

A fase de avaliação se refere à averiguação da aprendizagem, da qual o professor se utiliza para dar início à avaliação pedagógica. Sugere-se que ele promova um debate com os alunos a fim de verificar se conseguiram sistematizar e estruturar as ideias acerca do problema tratado no T-VILO. Ele deve também verificar se lograram êxito para destacar os conceitos e informações importantes sobre o conteúdo.

Com o fim de manter a qualidade dos produtos apresentados aos usuários, pode fazer parte de um cenário de aprendizagem, a avaliação da usabilidade da aplicação TVILO Viewer, usada tanto na TVDi como no celular. Essa avaliação pode ser feita pela equipe técnica, que mensura aspectos de desempenho dos usuários ao acessarem a aplicação, como percebidos pelos profissionais. Senão houver essa equipe, o professor pode ficar atento e fazer anotações sobre os comportamentos dos alunos, como a dificuldade deles para completar uma tarefa; a frequência de ajuda solicitada e o número de ações incorretas para um aluno atingir um objetivo de interação desejado.

\section{RESULTADOS ALCANÇADOS - CONTEÚDOS CRIADOS E CENÁRIOS DE APRENDIZAGEM DE MATEMÁTICA DEFINIDOS}

Nesta pesquisa, houve o envolvimento de dois produtores do conteúdo (pedagogos) e dois técnicos. Cinco T-VILOS foram desenvolvidos, os quais foram usados por alunos de duas escolas da rede pública de ensino com uma faixa etária de 9 a 12 anos de idade em experiências realizadas em três cenários de aprendizagem de matemática, como ilustra a Tabela 1.

O objetivo de cada experiência foi examinar os comportamentos dos usuários com relação aos usos dos T-VILOs apresentados. Além disso, observou-se a reação dos produtores de conteúdo ao desenvolverem cada T-VILO. 
Tabela 1 . Resumo dos T-VILOS desenvolvidos neste estudo

\begin{tabular}{|c|c|l|l|}
\hline Cenários & T-VILO & $\begin{array}{l}\text { Quantidade e especificação dos usuários } \\
\text { envolvidos }\end{array}$ & Conteúdos do T-VILO \\
\hline $\mathbf{1}$ & $\mathbf{1}$ & $\begin{array}{l}\text { 12 Crianças entre 9 -12 anos de idade que participam } \\
\text { de um projeto educativo de uma associação de } \\
\text { moradores. }\end{array}$ & $\begin{array}{l}\text { Operações básicas } \\
\text { matemática } \\
\text { (adição e subtração) }\end{array}$ \\
\hline $\mathbf{2}$ & $\mathbf{2}$ e $\mathbf{3}$ & $\begin{array}{l}\text { 10 Crianças entre 9 -14 anos de idade de uma Escola } \\
\text { do ensino fundamental e médio da rede estadual de } \\
\text { ensino. }\end{array}$ & Fração \\
\hline $\mathbf{3}$ & $\mathbf{4}$ e 5 & $\begin{array}{l}\text { 10 Crianças entre 9 -12 anos de idade de uma Escola } \\
\text { do ensino fundamental da rede municipal de ensino. }\end{array}$ & Fração \\
\hline
\end{tabular}

Os cenários de aprendizagem aconteceram em um laboratório de pesquisa, que possui uma sala de observação, onde os usuários podem ser observados ao interagirem com as aplicações transmitidas em tempo real. Os alunos foram sensibilizados para a tecnologia TVDi por um pedagogo, e em seguida divididos em grupos.

Os alunos em grupo deveriam acessar a aplicação interativa, que possuía conteúdos em um menu, e deveriam espontaneamente realizar outras ações para fazer uso dos T-VILOs. Essas ações poderiam ser: navegar nas mídias, acessar uma mídia, ler um texto, responder um questionário, dar sua opinião, etc. Quando um grupo terminava sua experiência, havia um grupo focal para que os alunos pudessem expressar seus sentimentos em relação aos momentos de aprendizagem vivenciados.

\section{ANÁLISE DOS RESULTADOS E IMPLICAÇÕES PARA 0 DESENVOLVIMENTO DE CONTEÚDOS EDUCATIVOS}

De uma forma geral, os resultados apontaram para a potencialidade da TVDi em suportar esta modalidade de ensino. Ao analisar as filmagens realizadas durante os cenários, autorizadas pelos responsáveis dos participantes, chegaram-se as reflexões que se seguem.

Os telespectadores aluno não tiveram dificuldades em navegar no conteúdo apresentado, depois que conseguiram acessar a aplicação, comportamento este esperado no uso de todos os T-VILOS. Eles ficaram motivados com a perspectiva de usar a TV como recurso de aprendizagem ou mesmo como material complementar às aulas escolares.

Constatou-se que eles inicialmente não estavam preparados para serem sujeitos ativos durante a experiência, ou seja, de buscarem interagir por eles próprios. Isto aconteceu em função do fato deles não estarem acostumados a interagir utilizando o controle remoto, e desta forma não perceberam as possibilidades de interatividade. Esta situação se modificou a partir da escrita do roteiro do T-VILO 4, cujo roteiro apresentou-se com uma comunicação bidirecional, bem diferente da perspectiva unidirecional dos anteriormente gerados. Nas modificações, o telespectador foi introduzido no desenvolvimento do enredo do vídeo, tornando-o parte integrante do processo de comunicação. Os alunos seguiram as orientações apresentadas no vídeo e perceberam a possibilidade de acessar a aplicação interativa disponível.

Em relação aos produtores do conteúdo, eles consideram que a metodologia pedagógica ajudou no planejamento dos conteúdos educativos. Mas houve necessidade de refazer os roteiros dos T-VILOS 2 e 3, de forma que o conteúdo atendesse a estrutura/dinâmica audiovisual de uma TV. Tal percepção foi ocasionada pelo fato de que, de uma forma geral, os telespectadores aluno tiveram dificuldades em realizar a convergência de mídias (áudio, vídeo e texto) proporcionada pela TVDi. Eles perceberam que os usuários não liam o que estava descrito na tela da TV, sendo assim não percebiam o que deveriam fazer para explorarem as suas potencialidades. 
Houve também uma análise do uso da tecnologia pelos produtores. O T-VILO 1 teve seu conteúdo feito pela equipe técnica, pois a aplicação T-VILO Maker ainda se encontrava em desenvolvimento. Para os T-VILOS 2 e 3, a aplicação T-VILO Maker já estava implementa e disponível na web, no entanto, os pedagogos que a utilizaram tiveram alguns problemas em relação ao uso e compreensão das funcionalidades disponíveis na interface da aplicação. Nos T-VILO 4 e 5 as práticas de produção foram bem cumpridas pelos profissionais envolvidos, em virtude da experiência adquirida nos primeiros T-VILOS.

\section{CONCLUSÃO}

Nesta pesquisa mostrou-se cenários que oportunizam estudos acerca da utilização da TVDi no âmbito educacional e de aspectos tecnológicos, comunicacionais e educacionais. Esses aspectos orientaram diversos profissionais como segue:

- A dimensão tecnológica orienta os profissionais na definição de possibilidades de obtenção de mais informações por meio de aplicações interativas, das quais o usuário navega e interage. Orientações tecnológicas propõem além do suporte à interatividade, favorecer a convergência de mídias para possibilitar o acesso e/ou a compreensão da informação por meio de outras mídias;

- A dimensão comunicacional guia os profissionais a promover uma maior participação do telespectador por meio de uma linguagem interativa. Essa categoria é expressa via conceitos trazidos no T-VILO: o apresentador de um vídeo e as interfaces das aplicações para TVDi que apresentam os conteúdos extras. A partir de orientações descritas neste artigo é possível pensar em uma linguagem interativa eficaz aplicada nos vídeos dos T-VILOs;

- A dimensão pedagógica auxilia os profissionais no planejamento e desenvolvimento de estratégias para que o telespectador possa construir seu próprio conhecimento, interferir no conteúdo transmitido, tornando-se um sujeito ativo durante o processo. A partir dos cenários de aprendizagem realizados, houve a evolução dos TVILOS ao longo deste estudo. Orientações foram descritas para auxiliar profissionais (desenvolvedores e pedagogos) a conhecerem as possíveis formas de interação com os materiais educacionais e a validarem aplicações e conteúdos produzidos no contexto da TVDi. Este conhecimento tende a contribuir para a construção de conceitos e metodologias pedagógicas que favoreçam o alinhamento entre a teoria e a prática educativa no universo das tecnologias digitais.

Como trabalhos futuros, pretende-se desenvolver uma estratégia de formação de profissionais a partir dessas três dimensões, a fim de desenvolver suas competências para atuar no desenvolvimento de conteúdos educativos. Pretende-se, também, inserir os próprios alunos como co-autores do conteúdo.

\section{REFERÊNCIAS BIBLIOGRÁFICAS}

ARBEX, Dafne F. ; SENS, André Luiz; SPANHOL, Fernando. TV Digital Interativa e EAD: a produção de conteúdos para programas educativos. Disponível em: http://lantec.fae.unicamp.br/tvdi/trabalhos/arbex.pdf. Acessado em: 08 de novembro de

2012.

BELDA, F. R. Um modelo estrutural de conteúdos para televisão digital interativa. Tese de Doutorado. Departamento de Engenharia de Produção, Universidade de São Paulo, 2010. 
BELDA, F. R.;DA SILVA, Roberta Salgado Gonçalves. Produção de conteúdo educativo em Televisão Digital como estratégia organizacional de comunicação universitária.

Disponível em: http://www.abed.org.br/congresso2008/tc/510200892151PM.pdf. Acessado em: 23 de janeiro de 2013.

BORGES NETO, Hermínio; SANTANA, J. Rogério. Sequência Fedathi: uma proposta de mediação pedagógica na relação ensino/aprendizagem. In: Vasconcelos, José Gerardo (Org.) Filosofia, Educação e realidade. Fortaleza: EUFC, 2003.

CAMPOS, Gerardo José. A televisão: objeto de ensino para uma educação de sujeitos: uma experiência em educação à distância. Fortaleza: LCR, 2005.

CASTRO, Cosette; FREITAS, C. Narrativa Audiovisual para Multiplataforma - Um Estudo

Preliminar. Disponível

em: http://www.intercom.org.br/bibliocom/sete/pdf/cosette-

castro-cristiana-freitas.pdf. Acessado em: 10 de maio de 2011.

CELES, Clayson Sandro F. de Sousa. Análise de aplicações convergentes para a televisão digital interativa baseada nos aspectos de sincronismo, portabilidade de conteúdo e da interação do usuário. Monografia de graduação (Graduação em Ciência da computação) - Universidade Estadual do Ceará (UECE). Fortaleza, 2011.

CRUZ, Renato. TV digital no Brasil: tecnologia versus política. São Paulo: Editora Senac São Paulo, 2008.

DE SOUZA, Clarisse Sieckenius. The Semiotic Engineering of Human-Computer Interaction. Cambridge. The MIT Press, 2005.

GINGA. Middleware Brasileiro Ginga. Disponível em: http://www.ginga.org.br/. Acessado em: 03 de junho de 2011.

JAKOBSON, R. Style in language, Ed. by T. Sebeok, Wiley New York, 2010.

KELLISON, Cathrine. Produção e direção para TV e vídeo: uma abordagem prática. Rio de Janeiro: Elsevier, 2007.

LISBOA, Rafaela Ponte; MENDES, Marília; FURTADO, Elizabeth; GUSSSI, Alcides; BORGES NETO, Hermínio. Requisitos para Elaboração e Integração de Conteúdos com Recursos da TV Digital Brasileira aplicados em Experiências de Aprendizado. Anais do XXI Simpósio Brasileiro de Informática na Educação, 2010.

LISBOA, Rafaela Ponte Lisboa. Interatividade e educação: os usos da metodologia pedagógica Sequência Fedathi na Televisão digital interativa. Dissertação (Mestrado) Faculdade de Educação, Universidade Federal do Ceará, Fortaleza, 2011.

LISBOA, Rafaela Ponte; FURTADO, Maria Elizabeth Sucupira; GUSSI, Alcides Fernando; BORGES NETO, Hermínio. Categorias Comunicacionais para Produção de Conteúdos Educativos para TVD. Anais do XXII Simpósio Brasileiro de Informática na Educação, 2011.

MATOS, Italo; FURTADO, Elizabeth. TvILOTool - Uma Ferramenta Orientada a

Serviços para Produção de Conteúdos para Televisão Digital Brasileira baseada em Objetos de Aprendizagem. Disponível em: http://luqs.unifor.br/wpcontent/uploads/2010. Acessado em: 10 de dezembro de 2012.

MATOS, Ítalo.T-VILO TOOL: ferramenta orientada a serviços para interação e produção de conteúdos educativos baseada no sistema brasileiro de televisão digital. Disponível em: http://www.tvdi.inf.br/site/artigos/Dissertacoes\%20(Mestrado)/TVILO\%20TOOL\%20-\%20SANTOS.pdf. Acessado em: 10 de dezembro de 2012.

ROESLER, Valter; BARBOSA, Maria L.K.; ARAUJO, Claúdio; WASSERMAN, F. BORDIGNON, A. Um novo modelo educacional através da TV Digital. RENOTE. Revista Novas Tecnologias na Educação, V. 8 No 2, julho, 2010. Disponível em: 
http://seer.ufrgs.br/renote/article/view/15254/9011. Acesso em: 25 de fevereiro de 2013.

SACRINI, M. Televisão digital interativa: expectativas de uso cultural e educativo. Comunicação \& Educação, São Paulo, v. Ano XI, p. 353-364, 2006. 